\title{
ESTADO ECOOLÓGICO DE ALGUNOS HUMEDALES COLOMBIANOS EN LOS ÚLTIMOS 15 AÑOS: UNA EVALUACIÓN PROSPECTIVA
}

\author{
Ecological status of some colombian wetlandsin the last 15 years: \\ a prospective evaluation
}

\author{
Karim Senhadji-Navarro1, Mauricio Andrés Ruiz-Ochoa² \& Juan Pablo Rodríguez Miranda ${ }^{3}$
}

Senhadji Navarro, K., Ruíz Ochoa, M.A. Rodríguez Miranda, J.P. (2017). Estado ecológico de algunos humedales colombianos en los últimos 15 años: Una evaluación prospectiva. Colombia Forestal, 20(2), 181-191.

Recepción: 20 de noviembre 2016

\section{Resumen}

Los humedales desempeñan un papel fundamental desde una perspectiva ecológica y socioeconómica. Frente a la idea ya obsoleta de lugares insalubres y de poca productividad, hoy son considerados ecosistemas estratégicos, amortiguadores de crecientes, sitios especiales de conservación de biota y cruciales por los servicios ecosistémicos que prestan. Partiendo de una revisión bibliográfica referente al estado ecológico de los humedales en Colombia en los últimos 15 años, se analizó cuáles son los factores, problemáticas e impactos más frecuentes y comunes que se presentan en 29 humedales colombianos, y se aplicó una metodología prospectiva, mediante el software MICMAC $®$, con el fin de conocer y priorizar los factores que son más sensibles a la transformación del ecosistema, y sobre los cuales habría que tomar acciones de mitigación, preservación y/o conservación. Se encontró que los procesos urbanísticos son un factor influyente de alto impacto en el deterioro del humedal, lo cual se obtuvo tanto con la revisión bibliográfica, como con el análisis y la priorización realizada con la prospectiva. Se pudo comprobar la potencialidad y robustez del software MICMAC ${ }^{\circledR}$ como herramienta
Aprobación: 22 de mayo 2017

de análisis prospectivo, lo cual podría ser usado por las autoridades ambientales para mejorar la toma de decisiones respecto a ¿Qué hacer?, ¿Cómo actuar? y ¿Qué esperar? de los factores y problemáticas que se presentan en los humedales. Por lo tanto, se podría disminuir el impacto ambiental generado sobre estos. Palabras clave: estado ecológico, humedales, MICMAC, priorización de acciones ambientales, prospectiva.

\begin{abstract}
Wetlands play a vital role from an ecological and socio-economic perspective. Faced with the idea outdated and unhealthy places and low productivity, today are considered strategic and crucial for ecosystem services provided by ecosystems, increasing dampers, special conservation sites biota. Based on a bibliographic review of the ecological status of wetlands in Colombia in the last 15 years, we analyzed the most frequent and common factors, problems and impacts presented in 29 Colombian wetlands, and a prospective methodology was applied through The MICMAC® software, in order to know and prioritize the factors that are most sensitive to the transformation of the ecosystem, and on which mitigation,
\end{abstract}

Unidades Tecnológicas de Santander. Bucaramanga, Colombia. knavarro@correo.uts.edu.co.

2 Unidades Tecnológicas de Santander. Bucaramanga, Colombia. mruiz@correo.uts.edu.co. Autor de correspondencia.

3 Facultad del Medio Ambiente y Recursos Naturales. Universidad Distrital Francisco José de Caldas. Bogotá, Colombia. jprodriguezm@udistrital.edu.co. 
preservation and/or conservation actions should be taken. It was found that the urban processes are an influential factor in high impact in the deterioration of the wetland, which was obtained both with the bibliographical revision, as with the analysis and the prioritization realized with the prospective one. The potential and robustness of the MICMAC $®$ software as a prospective analysis tool could be verified, which could be used by environmental authorities to improve decision making regarding What to do?, How to act?, and what to expect? Of the factors and problems that occur in wetlands. Therefore, the environmental impact generated on them could be reduced.

Keywords: ecological status, wetlands, MICMAC, priorization of environmental actions, prospective.

\section{INTRODUCCIÓN}

Los humedales, según la Convención de Ramsar en 1971, pueden ser entendidos como "aquellas extensiones de marismas, pantanos, turberas o aguas de régimen natural o artificial, permanente o temporal, estancado o corriente, dulce, salobre o salado, incluyendo las extensiones de agua marina cuya profundidad en marea baja no exceda de seis metros" (Secretaría de la Convención de Ramsar, 2013). Se encuentran entre los ecosistemas más productivos y diversos, con una alta prestación de servicios ecosistémicos (Herrera et al., 2008; Ruiz, 2014).

En Colombia, mediante la Ley 357 de 1997, se ratificó la Convención Ramsar y los humedales fueron reconocidos por su valor como ecosistemas estructurantes del territorio en diversas escalas temporales y geográficas por su integridad ecológica, de la que dependen la biodiversidad y procesos ecológicos como el del ciclo del agua y de nutrientes, entre otros (Herrera et al., 2008; Ruiz, 2014; Vilardy et al., 2014). Además, se tuvo en cuenta que los humedales hacen parte del acervo cultural del pueblo colombiano, por lo que la gestión y el manejo de estos ecosistemas está representada por los aspectos intrínsecos necesarios el desarroIlo sostenible del país (Herrera et al., 2008; Mosquera et al., 2015).

Las funciones ecológicas y ambientales de los humedales representan numerosos beneficios para la sociedad, pero en muchos casos el mal uso que se da a estas áreas genera degradación y pérdida del ecosistema (Secretaría de la Convención de Ramsar, 2013; Ruiz, 2014). Los humedales son afectados por diferentes factores, entre los que se encuentran: planificación y técnicas de manejo inadecuadas y políticas de desarrollos sectoriales inconsistentes y desarticuladas. Por lo anterior, Ruiz (2014) propone establecer estrategias de planificación y manejo integral para conocer los humedales y sus problemas, esto con el fin de fortalecer los procesos que actualmente se adelantan en ellos y divulgar las acciones emprendidas y por emprender para su protección y conservación.

Por su parte, la prospectiva es una forma de planificar y de encarar proyectos que están relacionados con un futuro "deseable" (Rodríguez, 2001), incluso algunos países han creado entidades específicas dedicadas a realizar estudios de prospectiva sobre diferentes aspectos que se proyectan hacia el futuro, tal como lo refieren Martín (1995) y Rodríguez (2001). En este sentido, se considera que el desarrollo de un territorio solo puede ser fruto del dinamismo endógeno, de ahí que sus procedimientos se basen en tres criterios de la prospectiva: anticipación, acción y apropiación (Farrés \& Toro, 2014).

Entre otros campos, la prospectiva se ha aplicado en marketing (Garza \& Cortez, 2011), sociología (Giménez, 2003), política (Martín, 1995; Medina et al., 2014) y pedagogía (Lizcano, 2012). Pero en ecología, y más específicamente en humedales, se encontraron pocos estudios reportados en la literatura; en este caso se tienen los trabajo de grado de Dueñas \&Contreras (2015) y Rodríguez \& Suárez (2017). Por otro lado, Ruiz (2014) realizó un trabajo 
de prospectiva, pero más que aplicar un método como tal lo que hizo fue describir el humedal y sus posibles respuestas en el futuro. Es decir, tal como lo mencionan Farrés \& Toro (2014) son errores que se cometen en la toma de decisiones asociados a: pensar como expertos en beneficio de la acción (el error tecnócrata, muy común en la praxis territorial moderna), y desechar a los expertos para dar la palabra al pueblo (el error populista, presente en bastantes intentos posmodernos de gestar procesos participativos). Sin embargo, en lo conceptual sí existe una temática relacionada con prospectiva ecológica, la cual tiene como objetivo deseado el desarrollo sustentable, lo cual se respalda en: 1) observar de otro modo (no fiarse de las ideas recibidas), 2) observar conjuntamente (apropiación), y 3) utilizar métodos tan rigurosos y participativos como sea posible para reducir las inevitables incoherencias colectivas (Farrés \& Toro, 2014). Estas técnicas están especialmente indicadas en contextos donde se requiere una amplia participación, y no requieren el uso de un aparato matemático muy sofisticado (Ambrosio-Albalá et al., 2011).

Lo innovador de este trabajo consiste en resaltar la importancia de aplicar la prospectiva en el campo de la ecología como una herramienta de análisis para elaborar escenarios futuros, a la hora de tomar decisiones en la gestión de los ecosistemas. Estos escenarios, según Martín (1995) y Ruiz (2014), tienen la facilidad de variar según la dinámica de las múltiples variables que estén presentes en los procesos antrópicos enlazados a las decisiones administrativas, en este caso de incidencia en el humedal, y al desarrollo de cualquier política ambiental. Por lo tanto, el futuro se fundamenta en técnicas que sirvan para estructurar procesos de deliberación colectiva, describir situaciones en el presente y elaborar visiones a largo plazo (Godet, 2001).

\section{MATERIALES Y MÉTODOS}

Partiendo de una revisión bibliográfica referente al estado ecológico de los humedales en Colombia en los últimos 15 años, inicialmente se analizó cuáles son los factores, problemáticas e impactos más frecuentes y comunes que se presentan en 29 humedales colombianos. Luego se aplicó una metodología prospectiva, mediante el software MICMAC $($ Gonod, 1996), con el fin de conocer y priorizar los factores que son más sensibles a la transformación del ecosistema y sobre los cuales habría que tomar acciones de mitigación, preservación y/o conservación.

La revisión bibliográfica se realizó a partir de estudios y trabajos de investigación localizados en catálogos bibliográficos de la Universidad Industrial de Santander (UIS), Google Academic, Jstor, Redalyc, Scielo, Scopus, Springer. El periodo de tiempo de análisis se estableció en razón a que: 1) el último Plan Nacional para el Manejo de las Aguas Residuales (PMAR) fue aprobado por el Consejo Nacional Ambiental en el año 2000, lo que de alguna manera influye en el estado actual de los humedales; y 2), en los últimos años ha existido un creciente interés en el estudio de estos ecosistemas estratégicos en el país. La información recogida sirvió para crear una base de datos estructurada en función de: año de publicación, localización del humedal, factores que estaban afectando al humedal, metodologías empleadas en el estudio, principales problemáticas, medidas correctoras que deberían aplicarse al humedal y estado ecológico del humedal.

Se utilizó el análisis prospectivo ya que se trata de un método el cual asume que el futuro no surge de extrapolar tendencias en el pasado y, por lo tanto, se puede construir definiendo escenarios deseables y viables e impulsando las acciones necesarias para alcanzarlos (Godet, 2001). Tal como lo describen Ambrosio-Albalá et al. (2011), el método describe un sistema identificando las relaciones de influencia (en lugar de las relaciones de causa-efecto) entre los elementos integrantes del sistema. Esta descripción se lleva a cabo por medio de un proceso de reflexión y deliberación colectiva en el que se rellena una matriz de doble entrada (Mendoza et al., 2011; Rodríguez, 2001), la 
cual permite generar unas jerarquías y clasificaciones de los elementos conforme a sus propiedades de motricidad (influencia que un elemento ejerce sobre otro) y dependencia (influencia que un elemento recibe de otro). De esta forma, el método resalta la estructura de las relaciones existentes entre las variables del sistema y señala cuáles son esenciales en su evolución.

La aplicación del método se llevó a cabo a través de la definición del sistema, de los subsistemas y de las variables, así como la evaluación de la matriz de influencia directa (MDI por sus iniciales en inglés; Godet, 2001). Es decir, se califica la influencia de variable $Y$ sobre la variables $X$ en un rango de 0 a 3, con las siguientes categorías: 0 ( $\sin$ influencia), 1 (influencia leve), 2 (influencia moderada) y 3 (influencia fuerte), los planos de desplazamientos, la priorización de las variables y la valoración de los factores críticos.

La información anterior fue utilizada para construir el sistema denominado "Humedal", el cual responde a un enfoque sistémico (figura 1) enfocado en el desarrollo sostenible, el cual tuvo como propósito identificar y definir las transformaciones de los humedales, respondiendo a preguntas como: ¿qué hacer?, ¿cómo actuar? y ¿qué esperar?

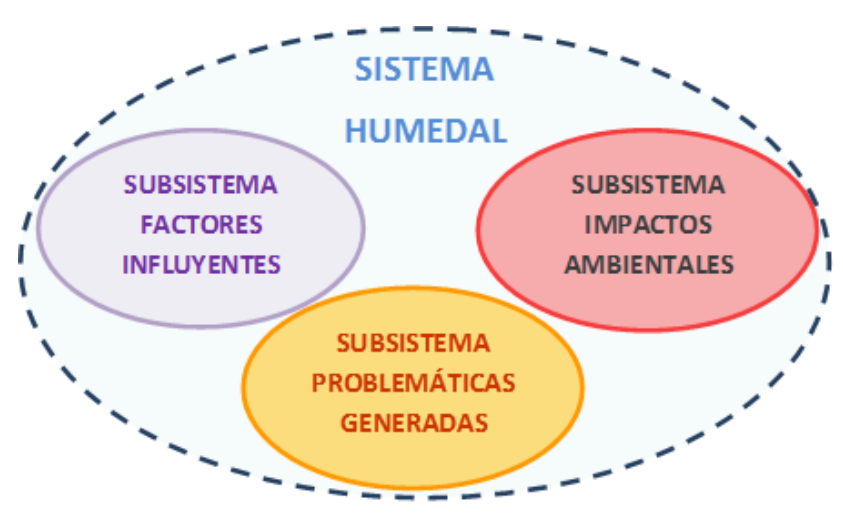

Figura 1. Esquema conceptual de la definición del sistema y los subsistemas. Fuente: adaptado de Dueñas \& Contreras (2015).

Para la valoración de los factores críticos se aplicó un método de priorización desarrollado por los profesores Marco Antonio Pereira y Mauricio Ruiz-Ochoa de las Unidades Tecnológicas de Santander (com. per), el cual tuvo como base el trabajo de grado realizado por Díaz \& Rincón (2015). Por lo tanto, el sistema "Humedal" conformado por tres subsistemas (figura 1) tuvo $n$ variables críticas cada uno, con lo que al final totalizaron las $N$ variables críticas del sistema. El método de priorización propuesto, se fundamentó en el cálculo del valor estratégico de las variables, el cual corresponde a la suma de los pesos de las proporciones de la influencia indirecta y de las proporciones de las dependencias indirectas dados por el software MICMAC®. Así, lo primero consistió en calcular el peso total del valor estratégico del sistema ( $P T_{s i s)}$ mediante la ecuación 1 :

$$
\text { PTsis }=\sum_{i=1}^{n} \text { PSub }(i)
$$

donde, PSub (i) corresponde al peso del valor estratégico del subsistema $i$, y $n$ representa el número de subsistemas.

Luego, se calculó el factor de peso del valor estratégico de cada subsistema $i$ (FpSub (i), ecu. 2):

$$
F p S u b(i)=\frac{P S u b(i)}{P T s i s}
$$

Posteriormente, a partir de la ecuación 2 se determinó el factor de contribución del valor estratégico de cada subsistema $i\left(F_{c} S u b(i)\right.$, ecu. 3):

$$
F c S u b(i)=\frac{F p S u b(i) * P V c S u b(i)}{P S u b(i)}
$$

donde, $P V_{c} S u b(i)$ corresponde al peso del valor estratégico de las variables críticas de cada subsistema $i$.

Con los cálculos de los factores de contribución obtenidos con la ecuación 3, se determinó el índice de criticidad (IC) del sistema (ecu. 4):

$$
I c=\sum_{i=1}^{n} F c S u b(i)
$$

donde, $i=1$, 2, y 3 , y $n$ representa el número de subsistemas. 
Finalmente, se determinó el factor de contribución de cada variable crítica en el sistema $\left(F_{C V}\right)$, a través de la ecuación 5:

$$
F c v=\frac{P v * F c S u b(i)}{P V c S u b(i)}
$$

donde, $P v$ representa el peso del valor estratégico de cada variable crítica. Con los resultados de la ecuación 5 se listaron todas las variables y se calculó el índice de priorización normalizado $\left(I P_{N}\right)$, el cual varía entre 0 y 100 . Para esto se aplicó la ecuación 6:

$$
I P_{N}=\left(\frac{F c v-F c v_{\min }}{F c v_{\max }-F c v_{\min }}\right) * 100
$$

donde, $F C v_{\text {min }}$ corresponde el valor mínimo del factor de contribución de cada variable crítica y $F_{C v_{\max }}$ es el valor máximo del factor de contribución de cada variable crítica. Para la visualización de los resultados de $I P_{N}$ se empleó el espectro de priorización, basado en la gama de colores establecidos en el ábaco de Régnier (tabla 1):

Tabla 1. Valoración, escala de colores y características de la variable al momento de priorizar.

\begin{tabular}{lll}
\hline Valoración & \multicolumn{1}{c}{ Escala } & \multicolumn{1}{c}{$\begin{array}{c}\text { Característica de la } \\
\text { variable }\end{array}$} \\
\hline Fuerte & $75 \leq \mathrm{IP}_{\mathrm{N}} \leq 100$ & Muy alta transformación \\
Moderado & $50 \leq \mathrm{IP}_{\mathrm{N}}<75$ & Alta transformación \\
Débil & $25 \leq \mathrm{IP}_{\mathrm{N}}<50$ & Mediana transformación \\
Ninguna & $0 \leq \mathrm{IP}_{\mathrm{N}}<25$ & Baja transformación \\
\hline
\end{tabular}

Por último, las variables se ordenaron de mayor a menor, quedando las de muy alta transformación en los primeros lugares.

\section{RESULTADOS}

\section{Factores, problemáticas e impactos más frecuentes}

Se encontró que el $76 \%$ de los humedales se ubican en la región Andina, el 14\% en la región Caribe,
$7 \%$ la región Orinoquia y el 3\% en la región Amazónica. Para determinar el estado ecológico de los humedales se trabaja principalmente muestreando flora (12 humedales) y fauna (9 humedales), con ello solo el humedal Costero Obregón (3.4\%) ubicado en el municipio de Guapi (Cauca) presenta condiciones favorables para albergar diferentes organismos de macro invertebrados acuáticos. En el resto de los humedales, debido las condiciones ambientales y ecológicas, se está en riesgo de perder sus bienes y servicios como humedal e incluso a desaparecer.

Dentro de los principales factores que afectan a los humedales se tienen en un $51.7 \%$ los procesos urbanísticos, en un $17.2 \%$ el vertimiento de aguas residuales, en un $13.7 \%$ la actividad agrícola y en un $10.3 \%$ el vertimiento de residuos sólidos. En cuanto a las problemáticas más frecuente la contaminación hídrica aparece en un $43.3 \%$, seguida de cambio en la dinámica hídrica, desecación del humedal y aparición de especies invasoras, todos estos con un porcentaje igual a $17.2 \%$. Y dentro los impactos ambientales producidos como consecuencia de los factores el más repetido es pérdida de fauna y flora, seguido de la pérdida de biodiversidad y anoxia en el humedal con 51.7\%, 17.3\% y $9.8 \%$ respectivamente. Así, asociados a los tres subsistemas presentados en la figura 1, se determinaron las variables asociadas a los mismos para ser analizadas mediante prospectiva (tabla 2).

\section{Análisis prospectivo}

Los resultados de la calificación de las 30 variables (factores influyentes: 10 variables, problemáticas generadas: 11 variables, e impactos ambientales: 9 variables) a través de la MID, en términos de influencia y dependencia de cada variable pueden ser observados en el plano de desplazamiento (figura 2). Se encontraron cuatro variables críticas: actividad agrícola (Ag), deforestación (De) y ganadería $(\mathrm{Ga})$, asociadas al subsistema Factores Influyentes; y procesos de eutrofización (Peu) del subsistema Problemáticas Generadas. La variable 
Tabla 2. Relación de las variables trabajadas con su respectiva descripción y asociadas al subsistema que pertenecen.

\begin{tabular}{|c|c|c|c|c|}
\hline N. ${ }^{\circ}$ & Nombre largo & $\begin{array}{l}\text { Nombre } \\
\text { corto }\end{array}$ & Descripción & Subsistema \\
\hline 1 & Actividad agrícola & $\mathrm{Ag}$ & $\begin{array}{l}\text { Corresponde a las prácticas realizadas por las necesidades de } \\
\text { demanda del mercado. }\end{array}$ & \\
\hline 2 & Deforestación & De & Se refiere a la tala de árboles para las actividades antrópicas. & \\
\hline 3 & Incremento de salinidad & Is & $\begin{array}{l}\text { Representa los cambios en una de las características químicas } \\
\text { del agua. }\end{array}$ & \\
\hline 4 & Ganadería & Ga & $\begin{array}{l}\text { Corresponde al pastoreo intensivo que se lleva a cabo dentro } \\
\text { del ecosistema. }\end{array}$ & \\
\hline 5 & $\begin{array}{l}\text { Materia orgánica en descom- } \\
\text { posición }\end{array}$ & Mo & $\begin{array}{l}\text { Corresponde a la materia orgánica que se degrada por acción } \\
\text { microbiana. }\end{array}$ & \\
\hline 6 & Procesos urbanísticos & $\mathrm{Pu}$ & $\begin{array}{l}\text { Representa los cambios continuos que se presentan en el uso } \\
\text { del suelo. }\end{array}$ & $\begin{array}{l}\text { Factores } \\
\text { Influyentes }\end{array}$ \\
\hline 7 & $\begin{array}{l}\text { Relleno para control de inun- } \\
\text { daciones }\end{array}$ & $\operatorname{Re}$ & $\begin{array}{l}\text { Corresponde al proceso antrópico que se realiza sobre el } \\
\text { humedal para contener las inundaciones. }\end{array}$ & \\
\hline 8 & Sedimentación & Se & $\begin{array}{l}\text { Se refiere a la acumulación por deposición de todos los sedi- } \\
\text { mentos transportados por el agua. }\end{array}$ & \\
\hline 9 & Vertimiento de aguas residuales & Var & $\begin{array}{l}\text { Representa las aguas que se disponen sobre el humedal pro- } \\
\text { veniente de las actividades humanas. }\end{array}$ & \\
\hline 10 & Vertimiento de residuos sólidos & $\mathrm{Vr}$ & $\begin{array}{l}\text { Consiste en la disposición final de los residuos generados por } \\
\text { la población sobre el humedal. }\end{array}$ & \\
\hline 11 & Afectación estructura del suelo & Aes & $\begin{array}{l}\text { Corresponde a la alteración de la forma particular en la que } \\
\text { se agrupan las partículas del suelo. }\end{array}$ & \\
\hline 12 & Aparición de especies invasoras & Asi & $\begin{array}{l}\text { Representa la aparición de especies que son capaces de des- } \\
\text { plazar y extinguir a otras autóctonas. }\end{array}$ & \\
\hline 13 & $\begin{array}{l}\text { Cambios drásticos en el equili- } \\
\text { brio halo-hídrico }\end{array}$ & Ceh & $\begin{array}{l}\text { Se refiere a la alteración de los cuerpos de agua por variación } \\
\text { en la salinidad. }\end{array}$ & \\
\hline 14 & $\begin{array}{l}\text { Cambios en la dinámica } \\
\text { hídrica }\end{array}$ & Cdh & $\begin{array}{l}\text { Corresponde a la afectación realizada sobre el cauce del } \\
\text { humedal. }\end{array}$ & \\
\hline 15 & Contaminación hídrica & $\mathrm{Ch}$ & $\begin{array}{l}\text { Representa el estado en el que termina el humedal por activi- } \\
\text { dades humanas. }\end{array}$ & Prob \\
\hline 16 & Desecación del humedal & $\mathrm{Dh}$ & $\begin{array}{l}\text { Hace referencia al estado del humedal luego de que las acti- } \\
\text { vidades antrópicas han sido excesivas. }\end{array}$ & \\
\hline 17 & $\begin{array}{l}\text { Desaparición de especies } \\
\text { pioneras }\end{array}$ & Dep & $\begin{array}{l}\text { Corresponde a la pérdida de las primeras especies resistentes } \\
\text { que colonizaron el humedal. }\end{array}$ & \\
\hline 18 & Erosión & $\mathrm{Er}$ & $\begin{array}{l}\text { Se refiere al desgaste que se produce en la superficie del } \\
\text { humedal por la acción de agentes externos. }\end{array}$ & \\
\hline 19 & Pérdida de profundidad & Prof & Representa la disminución del nivel del agua del humedal. & \\
\hline 20 & Procesos de eutrofización & Peu & Corresponde al exceso de nutrientes en el agua del humedal. & \\
\hline 21 & $\begin{array}{l}\text { Proliferación de especies opor- } \\
\text { tunistas }\end{array}$ & Eso & $\begin{array}{l}\text { Corresponde al aumento de las especies oportunistas relacio- } \\
\text { nadas con el deterioro del humedal. }\end{array}$ & \\
\hline 22 & $\begin{array}{l}\text { Alteración en los ciclos biogeo- } \\
\text { químicos y biológicos }\end{array}$ & Acb & $\begin{array}{l}\text { Corresponde a los cambios de los elementos químicos en el } \\
\text { compartimiento biótico y abióticos del humedal por acciones } \\
\text { antrópicas. }\end{array}$ & \\
\hline 23 & Anoxia en el humedal & Ahu & Representa la ausencia de oxígeno dentro del humedal. & \\
\hline 24 & Fragmentación del hábitat & Fra & $\begin{array}{l}\text { Corresponde al proceso por el cual el humedal va quedando } \\
\text { reducido a parches. }\end{array}$ & \\
\hline 25 & Insostenibilidad del humedal & In & $\begin{array}{l}\text { Representa la pérdida de los sistemas biológicos y producti- } \\
\text { vos que sostiene a las especies que allí viven. }\end{array}$ & \\
\hline 26 & Mala calidad del agua & Mca & $\begin{array}{l}\text { Representa los cambios en las características físico-químicas } \\
\text { y biológicas del agua. }\end{array}$ & $\begin{array}{l}\text { Impactos } \\
\text { Ambientales }\end{array}$ \\
\hline 27 & Pérdida de fauna y flora & Pff & $\begin{array}{l}\text { Corresponde a la pérdida de fauna y flora producto del dete- } \\
\text { rioro del humedal. }\end{array}$ & \\
\hline 28 & Pérdida de la biodiversidad & $\mathrm{Pbi}$ & $\begin{array}{l}\text { Hace referencia a la perdida de la diversidad biológica del } \\
\text { humedal. }\end{array}$ & \\
\hline 29 & Pérdida de resiliencia & $\operatorname{Pr}$ & $\begin{array}{l}\text { Representa la pérdida de la capacidad de recuperación del } \\
\text { humedal frente a las perturbaciones. }\end{array}$ & \\
\hline 30 & Pérdida del espejo del humedal & Peh & $\begin{array}{l}\text { Se refiere a la pérdida de una parte del área total que ocupa } \\
\text { el humedal. }\end{array}$ & \\
\hline
\end{tabular}




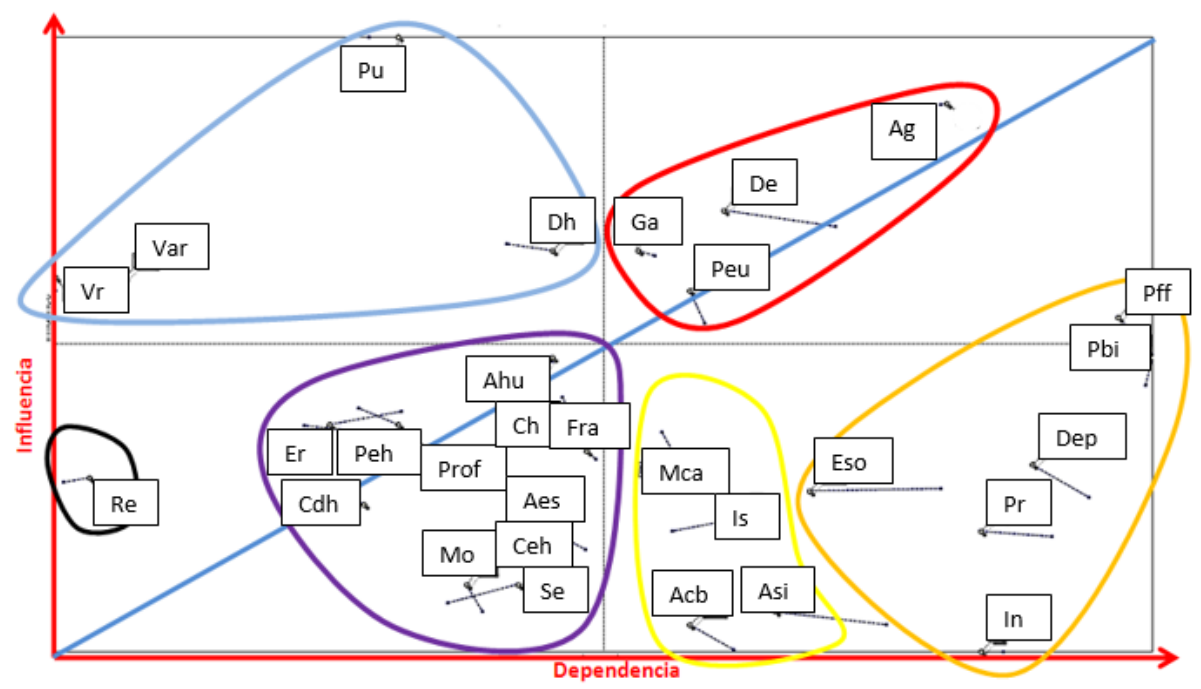

Figura 2. Plano de desplazamiento directo para las variables del sistema.

más influyente es procesos urbanísticos $(\mathrm{Pu})$, perteneciente al subsistema Factores Influyentes, mientras que la mayor dependencia consecuencia de esta influencia (impactos), estuvo dada por las variables pérdida de fauna y flora (Pff) y pérdida de biodiversidad (Pbi).

Como variables objetivo, y dado su nivel de dependencia, se encontraron seis variables distribuidas en dos subsistemas. Así, en el subsistema Problemáticas Generadas se tienen: desaparición de especies pioneras (Dep) y proliferación de especies oportunistas (Eso). Mientras, en el subsistema Impactos Ambientales se tienen: insostenibilidad del humedal (In), pérdida de fauna y flora (Pff), pérdida de la biodiversidad (Pbi) y pérdida de resiliencia (Pr). Por su parte, los resultados esperados (productos) de la dinámica del humedal están representados por las variables alteración en los ciclos biogeoquímicos y biológicos (Acb) y mala calidad del agua (Mca), asociadas al subsistema Impactos Ambientales; y aparición especies invasoras (Asi) e incremento de salinidad (Is) del subsistema Factores Influyentes.
Por su parte, 11 variables tuvieron un carácter predominantemente regulador materia orgánica en descomposición (Mo) y sedimentación (Se) del subsistema Factores Influyentes; afectación estructura del suelo (Aes), cambios drásticos en el equilibrio halo-hídrico (Ceh), cambio en la dinámica hídrica $(\mathrm{Cdh})$, contaminación hídrica $(\mathrm{Ch})$, erosión (Er), y pérdida de profundidad (Prof) del subsistema Problemáticas Generadas; y anoxia en el humedal (Ahu), fragmentación del hábitat (Fra) y pérdida del espejo del humedal (Peh) del subsistema Impactos Ambientales.

\section{VALORACIÓN DE LOS FACTORES CRÍTICOS QUE AFECTAN A LOS HUMEDALES}

En la tabla 3 se puede observar que los tres subsistemas tienen un factor de peso similar ( 0.3), y aunque el subsistema Problemáticas Generadas solo tuvo una variable crítica, fue el de mayor peso (0.36). Se identificaron cuatro variables críticas 
Tabla 3. Factores de peso y de contribución de los valores estratégicos para el sistema en estudio.

\begin{tabular}{lcccc}
\hline \multicolumn{4}{c}{ Subsistemas } \\
\cline { 2 - 5 } & Factores influyentes & Problemáticas generadas & Impactos ambientales & Total \\
\hline PTsis & 6140 & 7208 & 6621 & 19969 \\
FpSub & 0.31 & 0.36 & 0.33 & 1.00 \\
N. ${ }^{\circ}$ de var. críticas & 3 & 1 & 0 & 4 \\
PVcSub & 2655 & 771 & 0 & 3426 \\
FcSub & 0.13 & 0.039 & 0.00 & 0.17 \\
\hline
\end{tabular}

distribuidas así, donde tres pertenecen al subsistema Factores Influyentes: actividades agrícolas (Ag), deforestación (De) y ganadería (Ga), y una del subsistema Problemáticas Generadas: procesos de eutrofización (Peu). A su vez, el Ic del sistema se ubicó en $17 \%$.

Al priorizar mediante el $I P_{N}$ se pudo establecer que actividades agrícolas (Ag-100\%), deforestación (De-52\%), y ganadería (Ga-3\%) son factores que influyen en la transformación de los humedales de manera muy alta, alta y mediana, respectivamente (tabla 2). Entre tanto, los procesos de eutrofización (Peu-0\%) son una problemática con baja influencia en transformación de un humedal.

\section{DISCUSIÓN}

La información recogida sirvió para conocer el estado de transformación de estos ecosistemas, esta es la base para diseñar lineamientos para su gestión y manejo tal como lo reconocen Paredes (2010) y Mosquera et al. (2015). Además, los factores mayoritariamente antrópicos, las problemáticas a las que conducen estos y los impactos ambientales generados por los mismos son aspectos estudiados por Junk et al. (2013) y Jiménez et al. (2016), quienes mencionan que la alta presión ejercida sobre los humedales tiene incluso consecuencias en el cambio climático.

En la mayoría de los humedales estudiados para determinar su estado ecológico se realizaron muestreos de fauna y flora, superando en amplio número los análisis físico-químicos. A su vez, dentro de los factores que más repercuten en el deterioro del estado ecológico del humedal, se destacan los procesos urbanísticos. Así, según Paredes (2010) y Junk (2013), el aumento de la población y el desarrollo urbanístico está ocasionando fuertes transformaciones ecológicas que conducen al deterioro drástico o a la desaparición de los humedales. Otros factores que también aparecieron como relativamente frecuentes en la alteración de los humedales fueron el vertimiento de aguas residuales, la actividad agrícola, el vertimiento de residuos sólidos y la deforestación, compatible con lo encontrado por Armenteras \& Rodríguez (2014).

En cuanto a las problemáticas que se generan en los humedales como consecuencia del efecto de los factores la más frecuente es la contaminación hídrica, ocasionada entre otras cosas por el vertido de aguas residuales y de residuos sólidos. Sin embargo, otras problemáticas generadas con relativa frecuencia corresponden al cambio en la dinámica hídrica, a la desecación del humedal y la aparición de especies invasoras. De este modo, los problemas de contaminación y deterioro ambiental en los humedales coinciden con zonas y municipios con altos niveles de pobreza (Andrade et al., 2014; Estrada \& Moreno, 2014). Es más, el Instituto Von Humboldt menciona que, de los 1124 municipios colombianos existentes, el 97\% presentan área de humedal, de los cuales el 52\% no presenta acueducto; a su vez, el $67 \%$ de los hogares no poseen alcantarillado (Mosquera et al., 2015).

Dentro de los impactos ambientales más frecuentes en los humedales se tienen la pérdida de fauna y flora, seguido de la pérdida de 
biodiversidad, la anoxia en el humedal, o la mala calidad de las aguas. En consecuencia, cabe deducir que la parte biótica de los humedales es muy sensible a los cambios y es la principal afectada en los procesos de deterioro del humedal. Lo anterior indica que tanto la pérdida de fauna y flora y la pérdida de biodiversidad son los impactos más frecuentes en los humedales (Franco et al., 2013; Armenteras \& Rodríguez, 2014; Valencia \& Figueroa, 2014). En consecuencia, los procesos urbanísticos influyen de manera directa sobre las variables biológicas mediante la pérdida de individuos (fauna y flora), y la disminución de la diversidad y, por consiguiente, los humedales presentan menor resiliencia y mayor vulnerabilidad a las perturbaciones (Valencia \& Figueroa, 2014; Mosquera et al., 2015).

En el ejercicio del análisis de los resultados de la prospectiva se debe tener en cuenta la ubicación de las variables dentro de los cuatro cuadrantes del plano (figura 2). Según lo descrito por Ambrosio-Albaláet al. (2011) se puede visualizar la distribución de las variables en función de lo influyente y/o dependiente que sean. Así, las autoridades ambientales podrán responder las preguntas ¿qué hacer?, ¿cómo actuar? y ¿qué esperar? en términos de:

1. Desarrollar estrategias de mediano y largo plazo si las variables se ubican en el cuadrante I (parte superior izquierda), conocido como la Zona de Poder, cuya característica principal es que corresponde a una zona "altamente" influyente pero "poco" dependiente. En este caso, no se está bajo el control del sistema.

2. Desarrollar estrategias de muy corto plazo (acción inmediata) si las para las variables se ubican en el cuadrante II (parte superior derecha), debido a que representa la zona de conflicto. Este es un cuadrante "altamente" influyente pero también "altamente" dependiente y está bajo el control del sistema.

3. Desarrollar estrategias de largo plazo (acción no prioritaria) si las variables se ubican en el cuadrante III (parte inferior derecha), ya que los resultados dependen de las variables de los cuadrantes
I y II. Es una zona de resultados en la cual se presenta "baja" influencia pero "alta" dependencia y está bajo el control del sistema. Es decir, es el resultado de la acción de los factores y a través de las variables críticas se puede influir en la variable deseada para modificar la respuesta del sistema.

4. Desarrollar estrategias de monitoreo y seguimiento de largo plazo (no prioritarias) si las variables se ubican en el cuadrante IV (parte inferior izquierda) pues son variables del entorno y con poca influencia sobre el sistema. El cuadrante IV corresponde a una Zona de Falsos Problemas o Problemas Autónomos en la cual se presenta "baja" influencia y "poca" dependencia, es decir, las variables no están bajo el control del sistema.

Tras realizar la valoración de las variables críticas se obtuvo que la actividad agrícola y la deforestación parecen ser los factores de mayor afectación en los humedales y, por tanto, podrían ser los más regulables. Es decir, cuando los humedales que se encuentren sometidos a varios factores de afectación, y entre ellos los dos anteriores, se tendría que tomar acciones de protección y/o recuperación en términos del corto y medio plazo, tal como lo sugieren Ricaurte et al. (2012), Junk et al. (2013), Dueñas \& Contreras (2015) y Rodríguez \& Suárez (2017). Por su parte, las variables ganadería y procesos de eutrofización también podrían jugar un papel importante a la hora de minimizar el deterioro de los humedales (Valencia \& Figueroa, 2014; Dueñas \& Contreras, 2015; Rodríguez \& Suárez, 2017).

La aplicación de la metodología de análisis prospectivo ha sido muy poco utilizada en el contexto ecológico. Sin embargo, a partir de los trabajos de Dueñas \& Contreras (2015) y Rodríguez \& Suárez (2017) se pudo comprobar la potencialidad

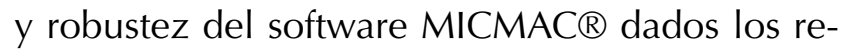
sultados obtenidos, en donde por ejemplo se encontró que en ambos casos la urbanización es una variable crítica a tener en cuenta en los procesos de conservación de los humedales. A su vez, con este análisis se pueden identificar las variables 
sobre las cuales se deben actuar de manera inmediata (variables críticas) y mejorar la toma de decisiones respecto a ¿qué hacer?, ¿cómo actuar? y ¿qué esperar? de los factores y las problemáticas a través de la disminución del impacto ambiental.

\section{CONCLUSIÓN}

Aunque la aplicación del método prospectivo en el contexto ecológico ha sido poco utilizada se pudo determinar dentro de los factores, problemáticas e impactos que afectan a los humedales cuáles son las variables que presenta mayor relevancia y qué tipo de estrategias (a corto, mediano y largo plazo) se deberían implementar para conocer el estado de transformación de estos ecosistemas. Por ejemplo, los procesos urbanísticos resultaron ser un factor influyente de alto impacto en el deterioro del humedal, lo cual se obtuvo tanto con la revisión bibliográfica como con el análisis y la priorización realizada con la prospectiva. A su vez, se comprobó la potencialidad y robustez del software MICMAC $®$ como herramienta de análisis prospectivo, el cual podría ser usado por las autoridades ambientales para mejorar la toma de decisiones respecto a ¿qué hacer?, ¿cómo actuar? y ¿qué esperar? de los factores y problemáticas que se presentan en los humedales. Así, se podría disminuir el impacto ambiental generado sobre estos.

\section{CONFLICTO DE INTERESES}

El autor declara no tener conflicto de intereses.

\section{CONTRIBUCIÓN POR AUTOR}

El autor único es responsable de la obra en todos los aspectos que condujeron a la elaboración de su publicación.

\section{REFERENCIAS BIBLIOGRÁFICAS}

Ambrosio-Albalá, M., Martín Lozano, J.M. \& Pérez Hernández, P.P. (2011). Aplicación del análisis estructural de prospectiva al diseño de estrategias de desarrollo rural: el caso de la comarca de Jerez. Estudios de Economía Aplicada, 29(1), 247-278.

Andrade, K.Y., Alturo, L.Y., Guerrero, N.Y. \& Lugo, L.J. (2014). Conflictos sociales y ambientales presentes en el humedal San Luis, Florencia (Caquetá, Colombia). Ingenierías \& Amazonia, 7(1), 48-55.

Armenteras, D. \& Rodríguez, N. (2014). Dinámicas y causas de deforestación en bosques de Latino América: una revisión desde 1990. Colombia Forestal, 17(2), 233-246.

Díaz, O. \& Rincón, S. (2015). Diseño de una metodología de evaluación cuantitativa de impactos ambientales en la construcción y operación de rellenos sanitarios en Colombia (Trabajo de pregrado, Ingeniería Ambiental). Bucaramanga (Colombia): Unidades Tecnológicas de Santander. 107 p.

Dueñas, N. \& Contreras, T. (2015). Revisión del estado ecológico de los humedales de Colombia en base a trabajos realizados en los últimos 15 años (Trabajo de pregrado, Ingeniería Ambiental). Bucaramanga: Unidades Tecnológicas de Santander. 74 p.

Estrada, L. \& Moreno, S.L. (2014). Análisis espacial de la pobreza multidimensional en Colombia a partir del censo de población de 2005. Revista ib, 3(1), 205-228.

Farrés, Y. \& Toro, F.J. (2014). La prospectiva estratégica como herramienta para la descolonización del saber territorial: una experiencia en Cuba. Revista de la Escuela de Arquitectura de la Universidad de Costa Rica, 1(5), 2-15.

Franco, L., Delgado, J. \& Andrade, G. (2013). Factores de la vulnerabilidad de los humedales altoandinos de Colombia al cambio climático global. Revista Colombiana de Geografía, 22(2), 69-85.

Garza, J.B. \& Cortez, D.V. (2011). El uso del método MICMAC y MACTOR análisis prospectivo en un área operativa para la búsqueda de la excelencia operativa a través del Lean Manufacturing. Innovaciones de Negocios, 8(16), 335-356. 
Giménez, G. (2003). El debate sobre la prospectiva de las ciencias sociales en los umbrales del nuevo milenio. Revista Mexicana de Sociología, 65(2), 363-400.

Godet, M. (2001). Creating futures. Scenario planning as a strategic management tool. Londres: Economica-Brookings Diffusion. $280 \mathrm{p}$.

Gonod, P. (1996). Dynamique des systèmes et méthodes prospective. Paris: Travaux et recherches de prospective, Futuribles International. $95 \mathrm{p}$.

Herrera, M.A., Sepúlveda, M.V. \& Aguirre, N. (2008). Análisis sobre la aplicabilidad de las herramientas de gestión ambienal para el manejo de los humedales naturales interiores de Colombia. Gestión y Ambiente, 11(2), 7-25.

Jiménez, A.M., Urrego, L.E. \& Toro, L.J. (2016). Evaluación del comportamiento de incendios de la vegetación en el norte de Antioquia (Colombia): Análisis del paisaje. Colombia Forestal, 19(2),161-180.

Junk, W.J. (2013). Current state of knowledge regarding South America wetlands and their future under global climate change. Aquatic Sciences, 75(1), 113-131.

Junk, W.J., An, S., Finlayson, C.M., Gopal, B., Kvêt, J., Mitchell, S.A. Mitsch, W.J. \& Robarts, R.D. (2013). Current state of knowledge regarding the world's wetlands and their future under global climate change: A synthesis. Aquatic Sciences, 75, 151-167.

Lizcano, D.L. (2012). Las dinámicas de desarrollo de la educación técnica y tecnológica en Santander mediante un estudio institucional. Revista Temas, 6, 73-96.

Martín, J.A. (1995). Prospectiva tecnológica: Una introducción a su metodología y a su aplicación en distintos países. Madrid: Fundación Cotec para la Innovación Tecnológica. 55 p.

Medina, J.; Becerra, S. \& Castaño, P. (2014). Prospectiva y política pública para el cambio estructural en América Latina y el Caribe. Santiago de Chile: Naciones Unidas. $338 \mathrm{p}$.

Mendoza, A., Quintero, I. \& Sarmiento, E. (2011). Aplicación de técnicas prospectivas. Ingeniare, 6(11), 25-36.
Mosquera, S.L., Nieto, O. \& Tapia, C. (2015). Humedales para la gente: visiones desde lo local. Bogotá D.C.: Instituto de Investigación de Recursos Biológicos Alexander von Humboldt. 98 p.

Paredes, D. (2010). Determinación de amenazas en humedales urbanos: Estudio de tres humedales de Valdivia, Chile. (Trabajo de pregado, Ingeniero en Conservación de Recursos Naturales). Valdivia (Chile): Universidad Austral de Chile. 35 p.

Ricaurte, L.F., Jokela, J., Siqueira, A., Núñez-Avellaneda, M., Marin, C., Velázquez-Valencia, A. \& Wantzen, K.M. (2012). Wetland Habitat Diversity in the Amazonian Piedmont of Colombia. Wetlands, 32, 1189-1202.

Rodríguez, J. (2001). Introducción a la prospectiva: Metodologías, fases y explotación de resultados. Economía Industrial, 6(342), 13-20.

Rodríguez, L. \& Suárez, S. (2017). Respuesta ecológica de dos humedales alto andinos ante cambios en sus factores físico-químicos y problemáticas asociadas (Trabajo de pregrado, Ingeniería Ambiental). Bucaramanga (Colombia): Unidades Tecnológicas de Santander. 54 p.

Ruiz, D.C. (2014). Análisis histórico y prospectiva del humedal Tierra Blanca. Perspectiva Geográfica, 19(1), 125-144.

Secretaría de la Convención de Ramsar. (2013). Manual de la convención de Ramsar: guía a la convención sobre los humedales (Ramsar, Irán, 1971). Gland: Secretaría de la convención de Ramsar. 120 p.

Valencia, P. \& Figueroa, A. (2014). Vulnerabilidad de humedales altoandinos ante procesos de cambio: tendencias del análisis. Revista Ingenierías, 14(26), 29-42.

Vilardy, S., Jaramillo, Ú., Florez, C., Cortés-Duque, J., Estupiñan, L., Rodríguez, J., Acevedo, O., Samacá, W., Santos, A., Peláez, S. \& Aponte, C. (2014). Principios y criterios para la delimitación de humedales continentales: una herramienta parafortalecer la resilencia y la adaptacion al cambio climatico en Colombia. Bogotá D.C.: Instituto de Investigación de Recursos Biológicos Alexander Von Humboldt. 100 p.

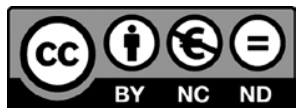

